\title{
RIEMANNIAN MANIFOLDS OF CONSTANT $k$-NULLITY ${ }^{1}$
}

\section{AARON ROSENTHAL}

1. Introduction. The purpose of this note is to derive curvature conditions that will guarantee the existence of a product structure for a Riemannian manifold of constant $k$-nullity. The proof is modeled after similar theorems for Riemannian and Kähler manifolds of constant nullity [5], [6]. Nullity was defined by Chern and Kuiper [1]. Ôtsuki defined the concept of nullity relative to a constant $k$, so that nullity became the special case $k=0$ [4]. A definition in terms of vectors was given by Gray, who also shortened the name to $k$-nullity [2].

2. Definitions and the main theorem. Let $M_{m}$ denote the tangent space to the Riemannian manifold $M$ at the point $m$, and let $R_{x y}$ denote the curvature transformation associated with $x, y \in M_{m}$.

Definition. Let $B_{x y} z=R_{x y} z-k\{\langle x, z\rangle y-\langle y, z\rangle x\}$, where $x, y, z$ $\in M_{m}$ and $k$ is a constant.

Then $B$ is a tensor of the same type as $R$, and $B$ possesses the symmetries of $R,[2]$.

Definition. Let $N_{k}(m)=\left\{z \in M_{m}: B_{x y} z=0\right.$ for all $\left.x, y \in M_{m}\right\}$.

$N_{k}(m)$ is called the $k$-nullity space at $m$. The dimension $\mu(m)$ of $N_{k}(m)$ is the $k$-nullity at $m$. The conullity space $C_{k}(m)$ is the orthogonal complement to the nullity space at $m$. Elements of $C_{k}(m)$ are called conullity vectors. A conullity plane is a plane spanned by conullity vectors.

TheOREM. Let $M^{n}$ be a complete, connected, and simply connected $C^{\infty}$ Riemannian manifold of constant $k$-nullity $\mu$, where $0<\mu \leqq n-3$. If $n-\mu$ is odd and the sectional curvatures of all conullity planes are unequal to $k$, then $M^{n}$ is a direct metric product, $M^{n}=K^{\mu} \times C^{n-\mu}$, where $K^{\mu}$ and $C^{n-\mu}$ are complete, and $K^{\mu}$ has constant curvature $k$.

3. Proof of the theorem. If $\mu$ is constant and positive, the distribution of $k$-nullity spaces is integrable, and the integral manifolds are complete submanifolds of $M^{n}$ of constant curvature $k,[2]$. Any one of these integral manifolds provides one factor for a product structure of $M^{n}$.

Definition. For each $u \in N_{k}(m)$ and $x \in C_{k}(m)$, let $T_{u}(x)=\mathrm{P}\left(\nabla_{x} U\right)$,

Received by the editors October 26, 1968.

1 This research was sponsored by the National Science Foundation under grant GP-8965. 
where $P$ is the projection of $M_{m}^{n}$ into $C_{k}(m)$ and $U$ is any nullity extension of $u$.

$\mathrm{T}_{u}$ is a well-defined linear operator on $C_{k}(m)$, called a conullity operator [6]. The nonvanishing of the conullity operators represents the obstruction to the existence of a product structure for $M^{n}$, for if each conullity operator is zero, we can apply DeRham's decomposition theorem to obtain the theorem [5].

LEMma (THE CONULLITY IDENTITY). If $T$ is a conullity operator at $m$, then

$$
\Im_{x, y, z} B_{x y}(T(z))=0 \quad \text { for all } x, y, z \in C_{k}(m) .
$$

Proof. Let $T$ be the conullity operator associated with $u \in N_{k}(m)$. The second Bianchi identity for $B$ states that $\Im_{x, y, z} \nabla_{x}(B)_{y z}(u)=0$. Using the definition of $B$ in terms of $R$, and the relation $\nabla_{X}(B)_{Y Z}(u)$ $=\nabla_{X}\left(B_{Y Z} u\right)-B_{\nabla_{X} Y, Z} u-B_{Y, \nabla_{X} Z} u-B_{Y, Z}\left(\nabla_{X} u\right)$, where $X, Y, Z$, and $U$ are extensions of $x, y, z$ and $u$, with $U$ a $k$-nullity field, we find that

$$
0=\Im_{x, y, z} B_{y z}\left(\nabla_{x} U\right)=\Im_{x, y, z} B_{y z}(T(x)) .
$$

Remark. Although this identity is valid for all values of $\mu$, it is nontrivial only when there are at least three independent conullity vectors. This is the reason for the $n-\mu \leqq 3$ hypothesis in the theorem.

LeммA. If $\lambda$ is a real eigenvalue of a conullity operator, then $\lambda$ is zero.

Proof. Let $T$ be the conullity operator at $m$ associated with $u \in N_{k}(m)$. We may assume that $u$ is a unit vector because $T$ is linear in $u$. As in Theorem (3.1) of [5], we calculate the curvature of $M^{n}$ along a unit speed geodesic $\sigma$ starting at $m$ in the $u$ direction. The frame field used in the calculation remains valid for this case [3]. If $P(t)$ is the matrix of $T_{\sigma^{\prime}(t)}$ relative to the adapted frame field used in this calculation, we obtain a differentiable matrix-valued function $P$ that satisfies the differential equation $P^{\prime}=-P^{2}-k I$. Since $M^{n}$ is complete, the domain of $P$ is the entire real line.

Let $x$ be an eigenvector of $P(0)$ with the real eigenvalue $\lambda$. The relation $P^{\prime}=-P^{2}-k I$ implies that $x$ is an eigenvector of any derivative of $P$ at time zero. Using the power series representation of $P$ given by Picard iteration, we can deduce that $x$ is an eigenvector of $P(t)$ for all $l$. Thus, we may assume that $P_{j 1}(t)=0$ for $j \neq 1$. If we set $p(t)=P_{11}(t)$, we find that $p$ satisfies the equation $p^{\prime}=-p^{2}-k$.

We can assume that $k \neq 0$, as this case is solved in Theorem (3.1) of $[5]$.

Thus, if $k<0, p(t)=\omega\left(p_{0}+\omega \tanh \omega t\right) /\left(\omega+p_{0}\right.$ tanh $\left.\omega t\right)$, where $\omega=\sqrt{ }-k$, and $p_{0}=p(0)$. 
If $k>0, p(t)=\omega\left(p_{0}-\omega \tan \omega t\right) /\left(\omega+p_{0} \tan \omega t\right)$, where $\omega=\sqrt{ }+k$.

In either case, if $p_{0} \neq 0$, the denominator of $p$ would vanish for some value of $t$, and $p$ would not be differentiable. Thus, $\lambda=p_{0}=0$.

To show that each conullity operator $T$ vanishes, it suffices to show that the eignevalues of $T$ are real and that $T$ can have no multiple eigenspaces with eigenvalue zero. The proofs of these facts are algebraic in nature, and are similar to Theorems (4.2) and (4.6) of [5], which used the conullity identity for $R$ and $T$, the symmetries of $R$, and the fact that the sectional curvatures of conullity planes were nonzero. In this case, we have the conullity identity for $B$ and $T$, the fact that $B$ shares the symmetries of $R$, and the fact that $\left\langle B_{x y} x, y\right\rangle \neq 0$ for all $x, y, \in C_{k}(m)$.

REMARK. It should also be clear that a theorem analogous to Theorem $\left(2^{*}\right)$ of [5] holds. That is, if we replace the hypotheses that $n-\mu$ is odd, and that the sectional curvatures of conullity planes are unequal to $k$, by the condition that the tensor $B$ is positive or negative definite when restricted to pairs of conullity vectors, then the conclusion of the theorem holds. This is again an algebraic consequence of the theorems in [5].

\section{REFERENCES}

1. S. S. Chern and N. H. Kuiper, Some theorems on the isometric imbedding of compact Riemann manifolds in Euclidean space, Ann. of Math. (2) 56 (1952), 422-430.

2. A. Gray, Spaces on constancy of curvature operators, Proc. Amer. Math. Soc. 17 (1966), 897-902.

3. B. O'Neill and E. Stiel, Isometric immersions of constant curvature manifolds, Michigan Math. J. 10 (1963), 335-339.

4. T. Ôtsuki, Isometric imbedding of Riemann manifolds in a Riemann manifold, J. Math. Soc. Japan 6 (1954), 221-234.

5. A. Rosenthal, Riemannian manifolds of constant nullity, Michigan Math. J. 14 (1967), 469-480.

6. - Kähler manifolds of constant nullity, Michigan Math. J. 15 (1968), 433440.

UnIVERSity of Colorado 